\title{
Performance assessment of Adaptive Optics techniques on FSO communications through the atmosphere
}

\author{
Noelia Martínez ${ }^{\mathrm{a}}$, Luis Fernando Rodríguez Ramos ${ }^{\mathrm{a}}$, Ángel Alonso $^{\mathrm{a}}$, and Zoran Sodnik ${ }^{\mathrm{b}}$ \\ ${ }^{a}$ Instituto de Astrofísica de Canarias (IAC),Vía Láctea s/n, San Cristobal de La Laguna, Spain \\ ${ }^{\mathrm{b}}$ European Space Agency, European Space Research and Technology Centre (ESTEC), \\ Noordwijk, The Netherlands
}

\begin{abstract}
Free Space Optical Communications have demonstrated in the recent years to be a feasible alternative to the radiofrequency links due to their advantages in terms of mass, power, cost and flexibility. Since 1995, the Optical Ground Station (OGS) at the Teide Observatory is used by ESA to test laser communication terminals on board of LEO and GEO satellites; however, as one side of the link is settled on the Earth surface, the communication performance will suffer from the effects of the atmosphere presence. The use of adaptive optics in both uplink and downlink, and even the use of Laser guide stars, will then contribute to improve link quality parameters. The approach of pre-correcting the uplink with a Deformable Mirror is also applicable to the Laser Guide Star self-propagating path and the fact a LGS will also narrow its beam with AO correction makes possible a more focused spot with lower magnitude up on the sky. With the aim of designing an Adaptive Optics system in the near future to improve the FSO links at the OGS and possibly the quality of LGSs, turbulence conditions regarding both uplink and downlink have been simulated. Expected performance of a proposed AO system to reduce atmospheric aberrations in both propagating directions is also analysed, as well as the application to LGSs and its advantages in comparison to conventional AO.
\end{abstract}

Keywords: FSO communications, Adaptive Optics, Uplink correction, Laser Guide Star

\section{INTRODUCTION}

A Free-Space Optical (FSO) communication system consists of a line-of-sight technology which transmits a modulated laser beam through the medium for broadband communications,$^{1}$ performed from satellite to satellite, from ground to ground, or from satellite to ground and vice versa. The field of interest at this point of the research is this last scenario: satellite-ground and ground-satellite FSO communications, known from now on as downlink and uplink, respectively.

As in this last case either the emitter or the receiver will be placed on the Earth's surface, both will suffer from the atmosphere influence no matter the direction in which the communication is performed. Turbulence (consequence of random refractive index fluctuations in the atmosphere itself) can severely impair uplink and downlink operation, although main effects on one link will differ from the other: considering a laser source placed in the deep space that spreads to hundreds or thousands of kilometres, its beam width exceeds by far the atmospheric characteristic spatial scales, therefore all the atmospheric eddies will be smaller and the main effects induced by them on the downlink will be those related to this idea: beam broadening, scintillation and loss of spatial coherence. However, an upward propagating laser, originated from an optical ground station and transmitted to space, has, at least with existing telescopes, a beam width smaller than the outer scale of the turbulence during all the atmospheric path. As a consequence, the uplink will be mostly affected by those phenomena large-eddies induced and will be steered by large angular displacements: beam wander and fluctuations in the angle-of-arrival. ${ }^{2}$

Therefore, a carefully designed and well behaving wavefront control in the optical links might improve the overall

Further author information: (Send correspondence to N.M.)

N.M.: E-mail: noelia@iac.es

L.R.R.: E-mail: lrr@iac.es 


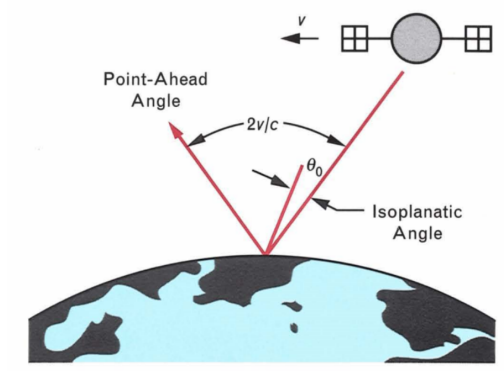

Figure 1: Geometry for propagating a laser beam to a satellite. The laser must be pointed ahead of the tracking direction by an angle of $2 v / c .^{3}$

communication performance, as this paper will try to demonstrate.

Adaptive Optics systems measure and correct the aberrations caused by the atmosphere and are extensively studied and used in astronomical ground observations. In this case, the incoming wavefront is measured by a wavefront sensor (WFS) and the image on sky corrected by a deformable mirror (DM); in the FSOC scenario, there is no image on sky to be improved but the received signal on the satellite (in the case of the uplink) and the deformable mirror shall pre-correct the beam before propagating it, adding more and more challenges to the design.

Furthermore, wavefront sensors need a reference close to the area of interest (a cosmic object for ground observations or the expected place where the satellite is going to be at the moment of the optical communication) in order to measure turbulence effects along these lines-of-sight and to use this measured signal for the deformable mirror control. In case of ground-based telescopes, it is unlikely to find a natural star bright enough to play this role; and obviously, the natural reference makes no sense for an optical link. The problem is solved then by using artificial references, so-called Laser Guide Stars (LGS).

Present paper is organised as follows: brief introduction to expose main factors affecting FSOC technology and AO systems, simulations of real scenario and expected performance for both applications (FSOC and LGS), and, finally, the summary of conclusions and future tasks to carry out in next development phases.

\subsection{Satellite Point-Ahead Geometry}

When propagating a laser beam to the spacecraft (uplink), the light shall travel to some distance at its specific velocity, $c$, and hence, this time delay in between the transmission and the reception will imply a displacement of the satellite inside its orbit, which will be translated into the so-called point-ahead angle.

The satellite point-ahead geometry (Figure 1) shall be taken into account not only for the pointing and tracking system, but also for AO purposes. As it has been mentioned before, the WFS needs a signal on sky to act as reference, although in case of uplink correction, the lack of one makes this sensing not possible and therefore, one reference shall be found: either an artificial star, or the downlink signal itself on the condition that both links are located inside the isoplanatic area (see next section) which will be strongly influenced by the point-ahead geometry depending on the speed of light and the spacecraft orbital velocity $(2 v / c){ }^{3}$

Geostationary and Low Earth orbits have been analysed to have knowledge of this parameter in each specific case: a geostationary satellite is characterized by a circular geosynchronous orbit in the plane of the Earth's equator with a radius of approximately $42,164 \mathrm{~km}$ measured from the center of the Earth; GEO satellites move with an orbital velocity $(v)$ of approximately $3.07 \mathrm{~km} / \mathrm{s}$; therefore, their point-ahead angle would be around $20 \mu \mathrm{rad}$ ( 4 arcsec). LEO orbiting devices have a velocity (v) of around $7.8 \mathrm{~km} / \mathrm{s}$ (reducing when increasing orbital altitude, which varies between $160 \mathrm{~km}$ and $2000 \mathrm{~km}$ above Earth's surface), therefore, their point-ahead angle would be approx. $50 \mu \mathrm{rad}(10 \mathrm{arcsec})$. 


\subsection{Atmospheric Isoplanatic Angle}

The isoplanatic angle defines a radius of a circle up on the sky within which the atmospheric wavefront disturbances may be considered approximately uniform. Isoplanatic angles are determined mainly by atmospheric turbulence at intermediate and higher altitudes. Two well-separated light waves (i.e. binary stars) travelling towards the telescope will tend to pass through separated turbulence structures, causing a decorrelation between the resulting aberration and imposing a limit on the isoplanatic angle; however, if the same light waves travelled only through lower altitudes, the turbulence structure would be the same as they are going through it and, therefore, aberrations would be basically equal.

Following the example of the binary star, the optical communication link travelling from one satellite to one ground station may suffer different turbulence conditions than the one travelling from the same location on Earth to the same satellite. The relationship between the isoplanatic angle on a specific location and the point-ahead angle difference of the downlink and the uplink has been studied and simulated in order to know if one link could be corrected with the wavefront measurements on the other one or, on the contrary, any other mechanism would be necessary to quantify and reduce the atmospheric influence like one Laser Guide Star launched to the sky point where the uplink will be pointed at.

\section{SIMULATIONS. PROCEDURES AND RESULTS}

With the aim of designing an Adaptive Optics system in the near future to pre-correct the uplink with a Deformable Mirror, turbulence conditions as well as the performance of an ideal uplink correction closed-loop have been simulated within the Object-Oriented Matlab Adaptive Optics toolbox (OOMAO), developed Conan and Correia in $2014 .^{4}$

The approach of pre-correcting the uplink with a Deformable Mirror is also applicable to the Laser Guide Star self-propagating path and the fact a LGS will also narrow its beam with AO correction makes possible a more focused spot up on the sky. Therefore, two study cases have been proposed: FSOC uplink correction and LGS uplink correction.

Regarding first study case, before analysing the system performance, it is necessary to simulate the isoplanatic patch at the Teide Observatory as it will have direct implication in the system design: the selection of a reference source for the adaptive optics system will depend on the results of this first type of simulation. In case of second application (LGS uplink propagation), the Laser Guide Star itself is assumed to be the reference object for the wavefront sensing (by pulsing the laser to allow measurements on axis avoiding back-scattering) in order to not depend on the availability of natural sources.

All simulations have been implemented within the Object-Oriented Matlab Adaptive Optics (OOMAO) Toolbox. It is a tool which has been designed for AO systems and recently, modified to add some functionality regarding the uplink correction research field. It is based on class objects: atmosphere class, telescope class, source class, wavefront sensor class, deformable mirror class and imager class. Each of them shall be set up with the proper parameters in order to be as representative of the real situation as possible.

Telescope has been modelled with two different sets of optical parameters: OGS Telescope at Teide Observatory, where FSO links take place, and WHT telescope at La Palma Observatory, where future LGS-Uplink correction experiments will occur (see Table 1).

The "ogsAtmosphere" function has been defined, within the "atmosphere class" framework, with the purpose of binding all OT atmosphere types together. The used atmospheric profiles have been extracted from Teide Observatory nocturnal profiles ${ }^{5}$ as well as from SHABAR measurements in case of diurnal analysis. Every data set has been integrated into the simulator by dividing the atmosphere distribution into four main layers; therefore, the available $C_{n}^{2}$ values have been used to calculate the global Fried parameter (corresponding to the atmosphere type under study) as well as the fractional Fried parameters related to each atmospheric layer. Global Fried parameter, $r_{0}$, has been computed with the expression in 1 , where $k$ is the wavenumber $k=2 * \pi / \lambda$; fractional Fried parameters, fro, have been calculated by the procedure described in $^{6}$ and summarised by the expression in 2.

$$
r_{0}=\left(0.423 k^{2} \int C_{n}^{2}(h) d h\right)^{-3 / 5}
$$


Table 1: Simulation Telescope Models

\begin{tabular}{|l|l|}
\hline \multicolumn{2}{|c|}{ Optical Ground Station (OGS) Telescope } \\
\hline Telescope Diameter & $1 \mathrm{~m}$ \\
\hline Optical configuration & Ritchey-Chrétien (RC) focus \\
\hline Focal Length RC & $13.3 \mathrm{~m}$ \\
\hline \multicolumn{2}{|c|}{ William Herschel Telescope (WHT) } \\
\hline Telescope Diameter & $4.2 \mathrm{~m}$ \\
\hline Optical configuration & Cassegrain focus \\
\hline Focal Length RC & $10.5 \mathrm{~m}$ \\
\hline
\end{tabular}

$$
f_{r_{0}}=\frac{C_{n}^{2}(h) \Delta h}{\int d h C_{n}^{2}(h)}
$$

The two different simulation procedures are described in the following section as well as their results concerning both study cases: first, studying the isoplanatic area at the Teide Observatory and second, examining the expected performance of an AO system for pre-correcting the uplink in both applications, optical communications and laser guide stars.

\subsection{Isoplanatic Area at the OT}

Analysing the isoplanatic area at the Teide Observatory has strong implications in the overall design, as in case downlink and uplink are located inside this patch, it will mean the measured wavefront in this downlink signal could be used as a reference source to pre-correct the uplink, whereas if not, an artificial reference would be fundamental for the proper performance of the AO system. In order to achieve these conclusions, the wavefront of both sources has been simulated with the available data (atmospheric profiles, telescope, sources) and it has been checked if each case study fulfils the isoplanatic condition of 1rad as maximum root-mean square difference of the two wavefronts (the one suffered by the downlink and the one suffered by the uplink). ${ }^{7}$

Communication links have been emulated within the OOMAO Toolbox by taking into account their expected location and the point-ahead angle between both, in such a way that the downlink has been simulated as a source at azimuth 0" and zenith 0", and the uplink as a source at azimuth 0" but zenith equal to the point-ahead angle in GEO and LEO satellites (4" and 10", respectively).

Results have been analysed by taking into account the main variables influencing this type of study: solar time and season when the atmospheric profiles were acquired. Additionally, the inferred conclusions are evaluated under the premise of the point-ahead angle related to each orbit case.

Notice that all simulation scenarios have been performed by placing the downlink at telescope zenith, and as it is well-known, this situation is the best one concerning amount of turbulence; if results are not satisfactory at this location, they will not be either at lower telescope elevations.

Figure 2 gathers the simulation results regarding the isoplanatic area at the OT. Seasonal and day lengths variations have been analysed; on one hand, Figures 2a and 2c (corresponding to nocturnal atmospheric measurements) show that, at GEO point-ahead angle (4") distance, the border of the isoplanatic patch is almost reached, meaning that the uplink may be corrected by the wavefront measurement on the downlink and that correction may be good enough. However, for the LEO case (10" point-ahead angle), both sources will be outside the isoplanatic area and correcting one with the incoming wavefront on the other one will not work out; in this last case, a Laser Guide Star, launched at the position where the uplink will take place, would be the best run to measure the turbulence the uplink will have to deal with. On the other hand, when dealing with diurnal links, the turbulence is much higher and concentrated at the ground layer, therefore, the isoplanatic patch becomes 


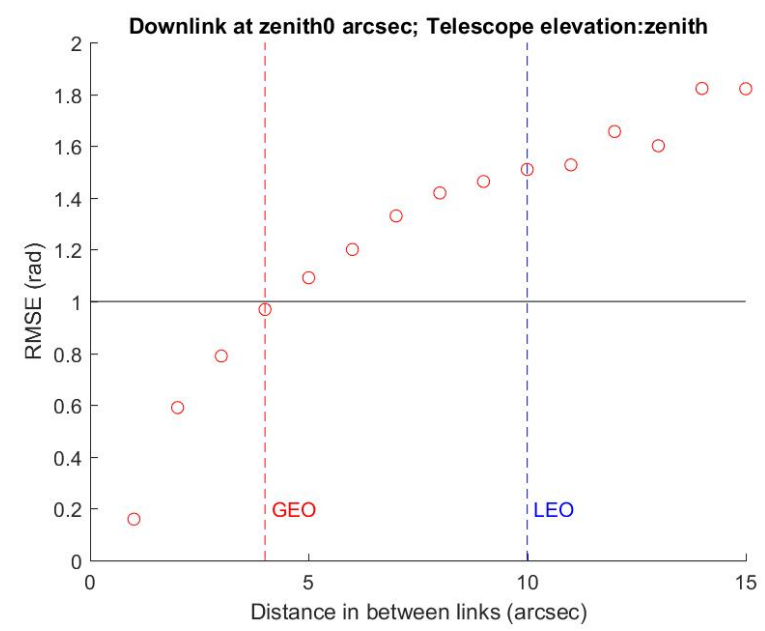

(a) Winter Night Profile

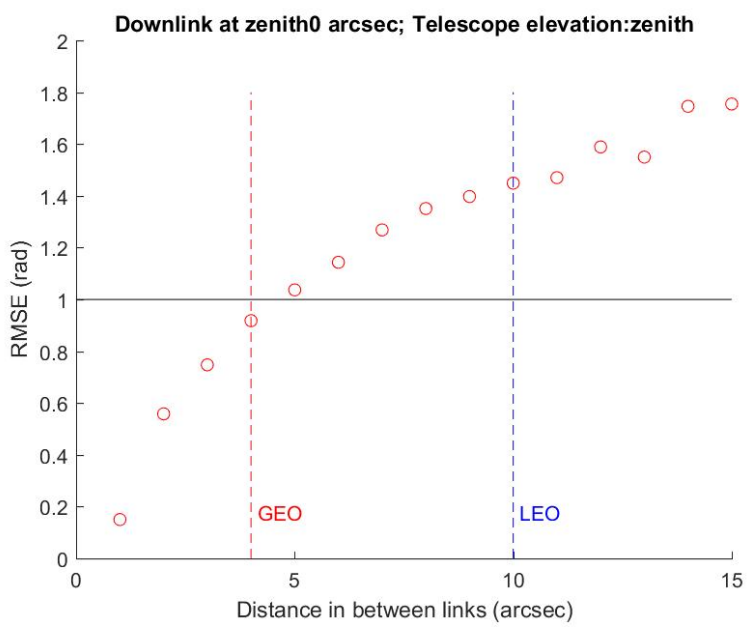

(c) Summer Night Profile

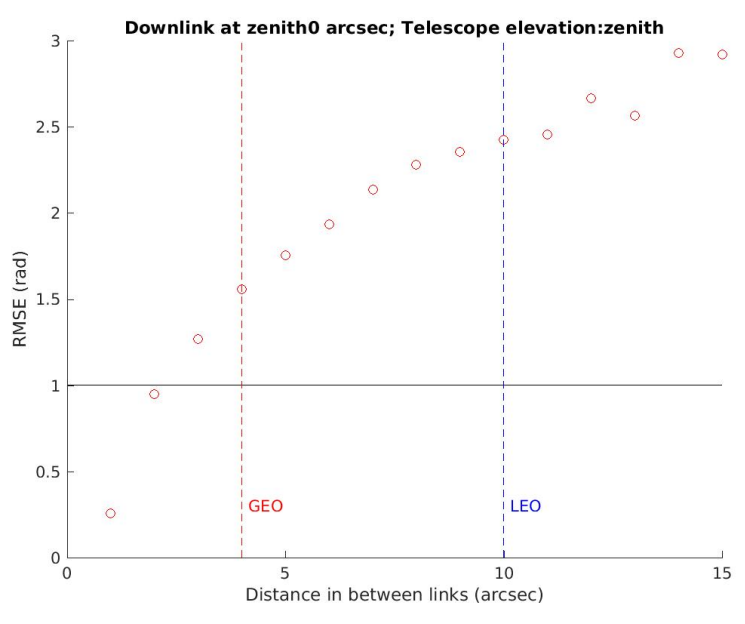

(b) Winter Day Profile

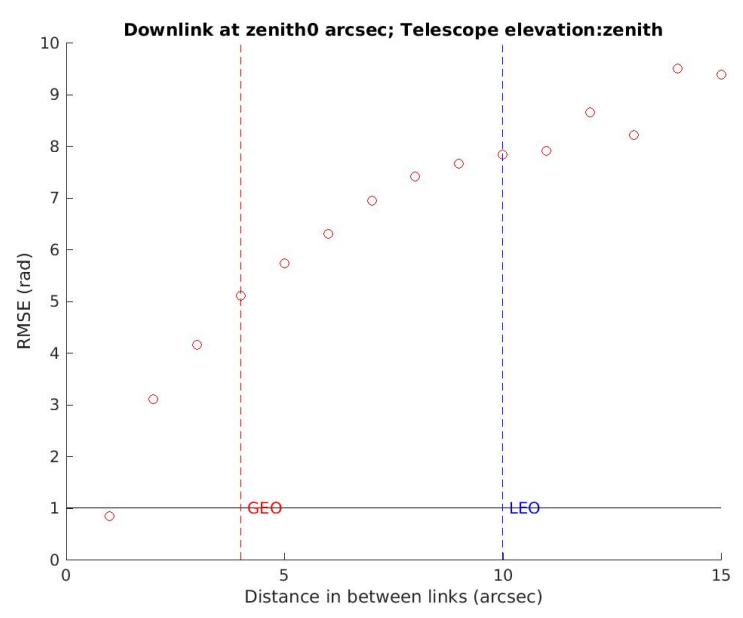

(d) Summer Day Profile

Figure 2: RMSE of the difference between phases of one downlink at telescope zenith and one uplink getting away 1" to 15" from zenith. Seasonal and day length variation analysis.

smaller. Figures $2 \mathrm{~b}$ and $2 \mathrm{~d}$ gather results of day-time simulations, pointing out the need of a LGS as a reference source for the uplink correction. Seasonal changes have also been analysed, although no big differences have been encountered as the OT location in the Canary Islands (Spain) has a quite stable temperature all year long. Conclusions of the isoplanatic area study remark the need of propagating a Laser Guide Star to the expected uplink location if an optimal uplink correction is pursued.

\subsection{Uplink Correction Performance}

A closed-loop simulation has been designed to evaluate the quality of pre-correcting the uplink in case of FSOC as well as for the self-propagating path of LGSs. In case of first application, the system performance has been assessed by using the wavefront measurements on the downlink itself to feed the deformable mirror and by sensing the atmosphere on an artificial star directly located at the expected location where the uplink would take place (separated from the downlink by the point-ahead angle: 10" in LEO satellites, considered the worst scenario). Metrics to analyse the performance of the uplink correction have been selected: the scintillation index (see expression 3) and the spot size of the beam at a given height over the Earth's surface (680km for the simulation), 
represented by the $D \sigma$ parameter (see expression 4) for the FSOC feeder link analysis; and only the spot size (as the scintillation has no interest in the conventional AO systems) at the Sodium layer (90-kilometre height over the ground).

Several diameters of launch telescope have been considered in order to cover different situations. For the communications application, diurnal and nocturnal atmospheric profiles have been used, in contrast to LGS precorrection, in which only nocturnal study case has been implemented as it is considered currently the most feasible scenario.

Each analysis has been performed 500 iterations.

$$
\begin{gathered}
\sigma_{I}^{2}=\frac{\left\langle I^{2}\right\rangle-\langle I\rangle^{2}}{\langle I\rangle^{2}} \\
D \sigma=\sqrt{\frac{\int_{-\infty}^{+\infty} \int_{-\infty}^{+\infty}\left(I(x, y)(x-\bar{x})^{2} d x d y\right)}{\int_{-\infty}^{+\infty} \int_{-\infty}^{+\infty} I(x, y) d x d y}}
\end{gathered}
$$

Figures 3 to 4 gathers the simulation results of the expected uplink correction performance when dealing with optical communications; and Figures 5 and 6 bring together the outcome of the LGS pre-correction simulations.

Regarding the predicted system performance in case of FSOC application, Figure 3 shows the simulation results when studying the scintillation index at a typical LEO height $(680 \mathrm{~km})$ and launching the laser from several telescope diameters. It is pointed out how correcting the uplink by the turbulence information on its location (propagating a LGS), would reduce the scintillation index in almost two orders of magnitude in the case of a winter night atmosphere (Figure 3a) and, even in a very turbulent scenario (diurnal atmosphere), the improvement is also clear when using smaller transmitter telescopes $(D<2 m)$ (Figure 3b). With respect to the other parameter of interest, the spot size up on the sky at the given height, results are gathered in Figure 4. Measurements are shown in equivalent arcseconds seen by the telescope. In case of nocturnal links (Figure 4a), the improvement in the performance is represented by a reduction of 0.2 " when using the LGS as a reference source, whereas there is no much difference when correcting the uplink with the downlink information. However, in diurnal scenarios it is when the performance improvement becomes more noticeable, with a reduction in the spot size of 1.2" (see Figure 4b) when propagating the artificial reference to the expected uplink location.

Concerning an AO pre-correction system for the self-propagation path of a Laser Guide Star, the only metric to be considered in the analysis has been the spot size at a 90-kilometre layer over the Earth's surface (Sodium Layer), as the scintillation index would not have an implication in the overall AO performance. Two scenarios have been taken into account: launching the laser through a 1-meter telescope (OGS) and through a 4.2-meter telescope (WHT), optical parameters of both telescopes have been described in previous section. Figure 5 presents the simulation results corresponding to the first case: the spot up on the sky would reduce its size in almost 50\%; when propagating the beam through a larger telescope (Figure 6) the improvement also increases due to the focusing capability of the telescope itself.

\section{CONCLUSIONS AND FUTURE DEVELOPMENT}

Two different types of simulations have been performed in order to, first, get knowledge about the isoplanatic area at the Teide Observatory, in both diurnal and nocturnal scenarios all year long, and, second, evaluate the expected performance of an adaptive optics system to pre-correct the feeder link in free-space optical communications and, on a separate issue, to pre-correct the self-propagating path of a Laser Guide Star, artificial reference in conventional AO. 


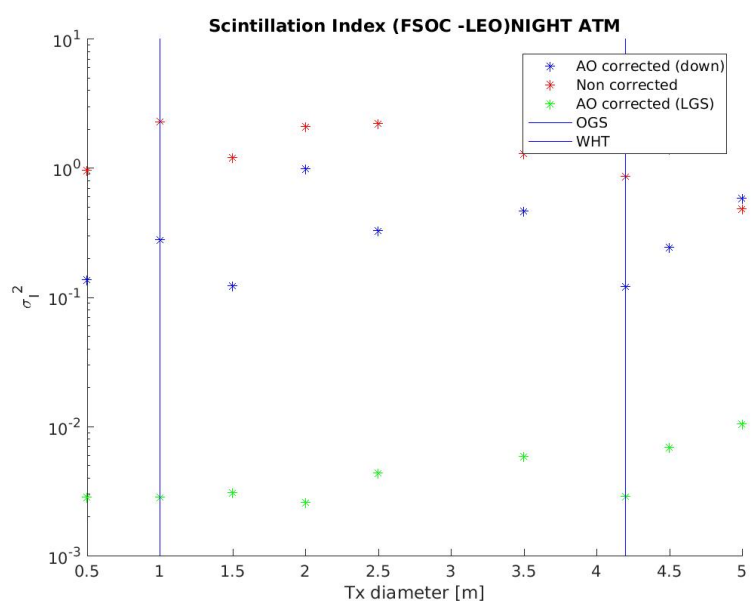

(a) Nocturnal Atmospheric Profile

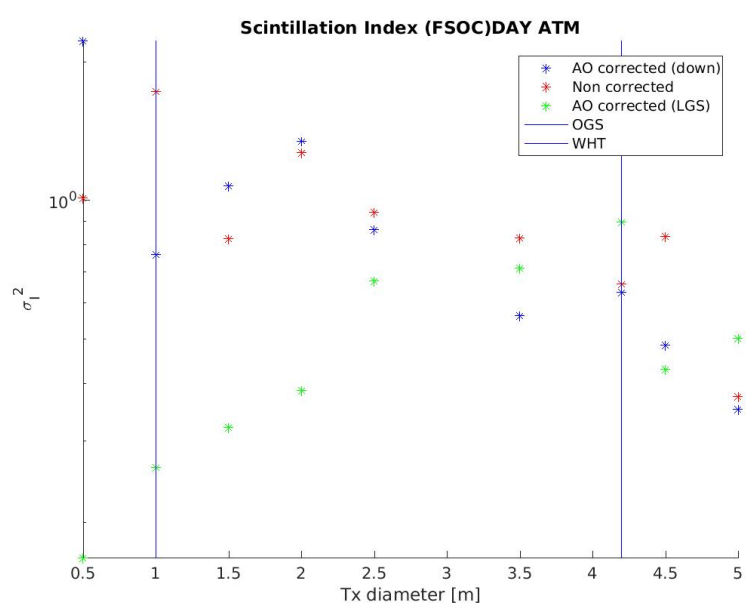

(b) Diurnal Atmospheric Profile

Figure 3: Uplink scintillation index with no AO correction (red), corrected by the measurements on the downlink position (blue) and by the measurements on the uplink location (green). Uplink and downlink are separated by 10" (LEO Satellite). Day length variation analysis.

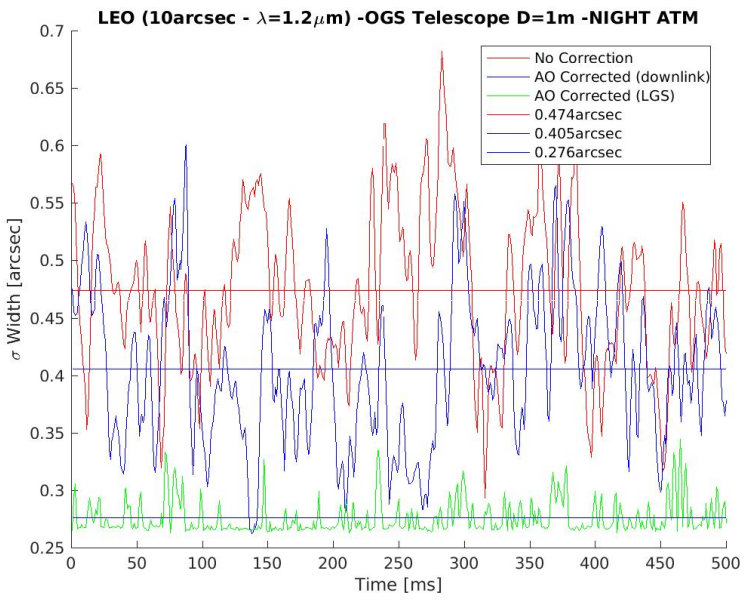

(a) Nocturnal Atmospheric Profile

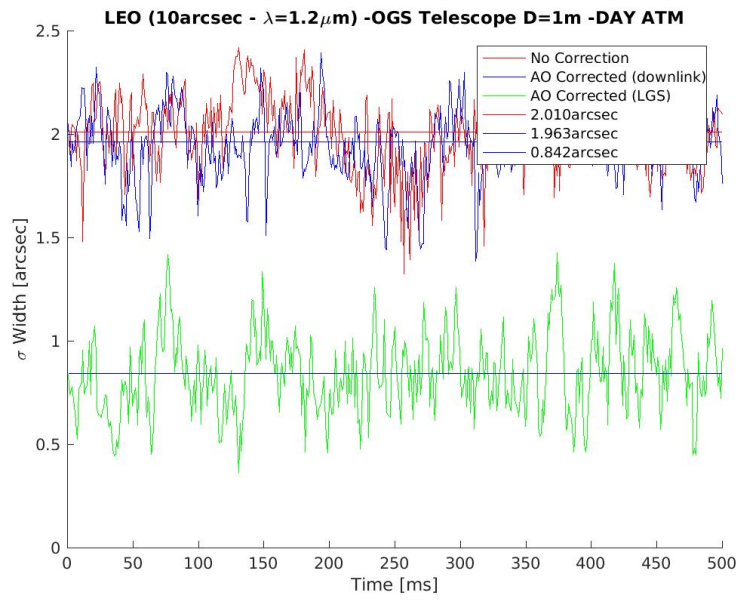

(b) Diurnal Atmospheric Profile

Figure 4: Uplink spot width with no AO correction (red), corrected by the measurements on the downlink position (blue) and by the measurements on the uplink location (green). In this case, uplink and downlink are separated by 10" (LEO Satellite). Day length variation analysis.

On one hand, regarding the isoplanatic study at the OT, due to the fact downlink and uplink are separated by a point-ahead angle on the sky (because of the satellite movement and the light traveling through a certain path), both links are proven to not be inside the isoplanatic patch; therefore the use of a LGS to measure the wavefront information on the uplink location is necessary in order to correct the higher modes of the turbulence.

On the other hand, concerning the uplink correction performance, scintillation index and spot size have been analysed in the FSOC case, showing large improvements when correcting the uplink by measuring turbulence on its location; in the LGS scenario, only the spot size has been considered as a parameter of interest, also establishing the improvement in the beam size after the uplink correction has been accomplished. 


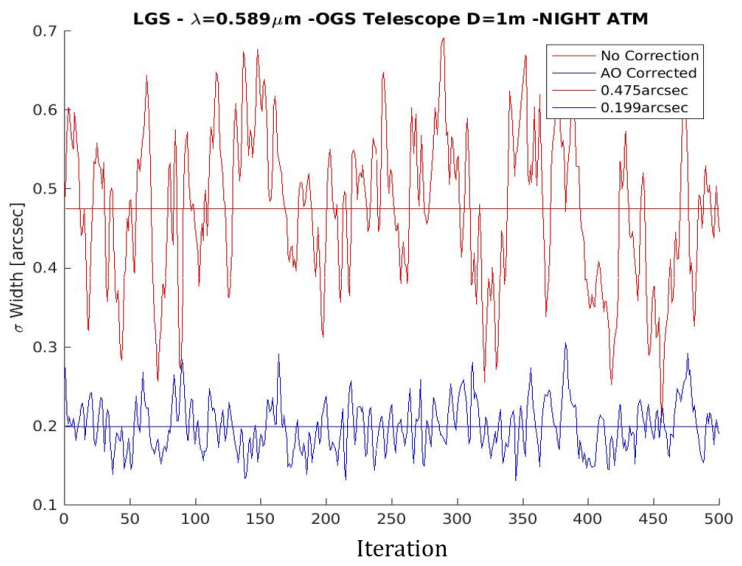

Figure 5: Uplink spot width with no AO correction (red) and corrected by the measurements on the LGS itself (blue). Beam launched by a 1-meter telescope.

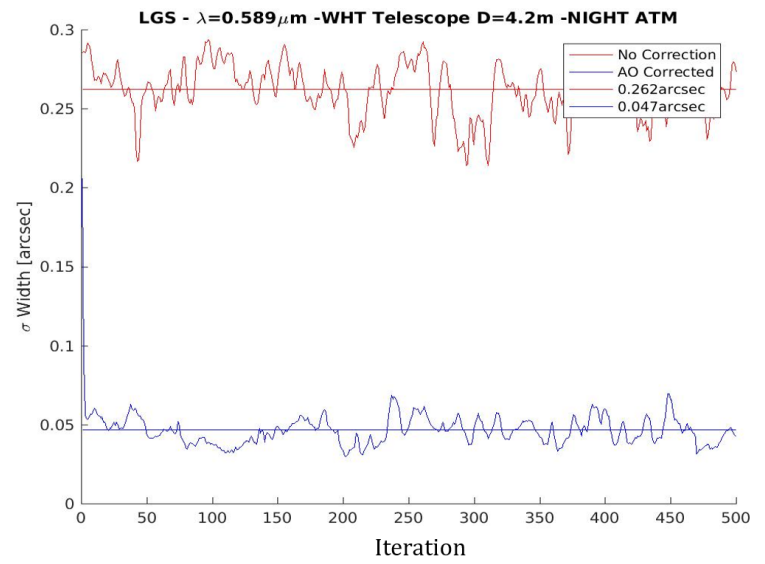

Figure 6: Uplink spot width with no AO correction (red) and corrected by the measurements on the LGS itself (blue). Beam launched by a 4.2-meter telescope. 
Future tests at the OGS in Tenerife (Spain) are planned to be performed during last quarter of the year, as well as experiments regarding uplink correction on Sodium Laser Guide Stars self-propagating path in collaboration with the European Southern Observatory (ESO) in the near future.

\section{ACKNOWLEDGMENTS}

The authors would like to thank the Instituto de Astrofísica de Canarias (IAC) and the European Space Agency (ESA) for co-funding this research, as well as Carlos Correia for the support and maintenance of the OOMAO simulator.

\section{REFERENCES}

[1] Raj, A. A. B., Selvi, J. A. V., and Raghavan, S., "Terrestrial free space line of sight optical communication (tfslsoc) using adaptive control steering system with laser beam tracking, aligning and positioning (atp)," in [Wireless Communication and Sensor Computing, 2010. ICWCSC 2010. International Conference on], 1-5 (Jan 2010).

[2] Hemmati, H., [Deep space optical communications], Wiley (2006).

[3] Parenti, R. R., "Adaptive optics for astronomy," The Lincoln Laboratory Journal 5(1), 93-114 (1992).

[4] Conan, R. and Correia, C., "Object-oriented matlab adaptive optics toolbox," (2014).

[5] García-Lorenzo, B. and Fuensalida, J. J., "Statistical structure of the atmospheric optical turbulence at teide observatory from recalibrated generalized scidar data," Monthly Notices of the Royal Astronomical Society 410(2), 934-945 (2011).

[6] Conan, R., [Object-Oriented Matlab Adaptive Optics. User Guide] (2013).

[7] Glindemann, A., [Principles of Stellar Interferometry], Springer (2011). 